\title{
Commentary on: Comparison of complication rates related to male urethral slings and artificial urinary sphincters for urinary incontinence: national multi-institutional analysis of ACS-NSQIP database
}

\author{
M. Horstmann ${ }^{1,2}$
}

Received: 25 June 2016 / Accepted: 28 June 2016 / Published online: 9 July 2016

(C) Springer Science+Business Media Dordrecht 2016

Both the artificial urinary sphincter (AUS) and male slings (MS) can be used for the treatment of male urinary stress incontinence. Whereas the AUS represents the gold standard and is indicated in moderate to severe incontinence, male slings are more often applied in mild to moderate incontinence [1]. In the present work, the authors compare complication rates of both procedures $(n=1205)$ that were prospectively gathered from a large independent multiinstitutional database. Even though the paper provides no functional outcome data, does not analyze specific urologic complication and does not differentiate between different types of male slings, it still contains one important message for patient counseling, namely that both the AUS and MS procedures are safe and have a low 30-day complication rate (AUS: $5.1 \%$ vs. MS: $2.8 \%$ ). This message is noteworthy especially because patients analyzed were high of age, mostly obese and had important comorbidities (see
Table 1). However, because of its drawbacks the paper cannot be used to individually counsel patients, but it provides some excellent data for patients to motivate them for the regionally still underused surgical treatment of male urinary stress incontinence [2]. Because of this message, this work is important and the authors should be applauded for their work.

\section{References}

1. Comiter CV, Dobberfuhl AD (2016) The artificial urinary sphincter and male sling for postprostatectomy incontinence: which patient should get which procedure. Investig Clin Urol 57(1):3-13

2. Liu JS, Hofer MD, Milose J, Oberlin DT, Flury SC, Morey AF, Gonzalez CM (2016) Male sling and artificial urethral sphincter for male stress urinary incontinence among certifying American Urologists. Urology 87:95-99
M. Horstmann

marcushorstmann@gmx.ch

Department of Urology, University of Jena, Jena, Germany

2 Malteser Hospital St. Josefshospital, Uerdingen, Krefeld, Germany 